\section{Hodgkin-Lymphom: Drei Regimes im Vergleich}

\author{
Kombinationschemotherapien bestimmen seit 20 Jahren die Behandlung \\ des frühen Hodgkin-Lymphoms. Bei zusätzlichen Risikofaktoren muss man \\ genauer hinsehen. Ein Vergleich dreier bekannter Varianten.
}

E ur Hodgkin-Lymphome (HL) im Frühstadium sind seit 20 Jahren Kombinationschemotherapien Standard. Welches Regime für welche Subgruppe das beste ist, wird kontrovers diskutiert. In der H9-U-Studie der EORTC wurden 3 verschiedene Regimes, gefolgt von einer Involved-field-Radiotherapie (IFRT), bei 808 Patienten mit zuvor unbehandeltem supradiaphragmatischem HL im Stadium I-II und mindestens einem Risikofaktor ( $\geq 50$ Jahre, Befall von 4-5 Lymphknotengebieten, Mediastinum/ Thorax-Quotient $\geq 0,35$, Erythrozytensedimentationsrate $[\mathrm{ESR}] \geq 50$ ohne oder $\geq 30$ mit B-Symptomen) verglichen. Die Grenze für Nichtunterlegenheit wurde definiert als 10\%iger Unterschied zwischen den geschätzten ereignisfreien Überlebensraten (EFS) nach 5 Jahren.
Zu Beginn der Studie Ende der 1990erJahre galten 6 Zyklen ABVD (Doxorubicin, Bleomycin, Vinblastin und Dacarbazin) als Standard. Dagegen wurden randomisiert 2 experimentelle Regimes getestet: 4 Zyklen ABVD (4-ABVD) sowie BEACOPP baseline (Bleomycin, Etoposid, Doxorubicin, Cyclophosphamid, Vincristin, Procarbazin, Prednison). Auf alle Regimes folgte eine IFRT.

Die EFS-Raten nach 5 Jahren lagen bei $85,9 \%$ für 4 -ABVD und bei $88,8 \%$ für 4-BEACOPP baseline $_{\text {und waren den } 89,9 \%}$ im Kontrollarm damit definitionsgemäß nicht unterlegen. Die Rate für das geschätzte 5-Jahres-Gesamtüberleben lag bei $94 \%$ (6-ABVD), $93 \%$ (4-ABVD) bzw. $93 \%$ (BEACOPP), für das 10-Jahres-Gesamtüberleben bei $93 \%$ (6-ABVD), $91 \%$ (4-ABVD) bzw. $89 \%$ (BEACOPP).
Die Patienten der BEACOPP-Gruppe entwickelten allerdings im Vergleich zu Patienten der beiden ABVD-Gruppen häufiger schwere Nebenwirkungen.

Fazit: Die Studiendaten zeigen, dass 4 Zyklen ABVD, gefolgt von IFRT, ein frühes HL mit Risikofaktoren gut kontrollieren. Auch wenn 4 Zyklen BEACOPP dem ABVD nicht unterlegen waren, kam es darunter doch zu mehr Nebenwirkungen. Seit Studienbeginn hat sich allerdings viel getan: Die deutsche Hodgkin-Studiengruppe nutzt inzwischen eine Mischung aus BEACOPPund ABVD-Zyklen; auch wird zur Risikostratifizierung und weiteren Therapieplanung zunehmend die Positronenemissionstomografie eingesetzt.

Christina Berndt

Fermé $C$ et al. ABVD or BEACOPPbaseline along with involved-field radiotherapy in early-stage Hodgkin Lymphoma with risk factors: Results of the European Organisation for Research and Treatment of Cancer (EORTC)eGroupe d'Etude des Lymphomes de l'Adulte (GELA) H9-U intergroup randomised trial. Eur J Cancer. 2017;81:4555.

\title{
Die Bestrahlung aller Lymphknoten bei HL
}

\section{Beim Hodgkin-Lymphom werden die betroffenen Lymphknoten oft gezielt mit einer Involved-Field-Radiotherapie (IFRT) bestrahlt. Häufig entstehen aber Rezidive an anderen Lymphknoten. Welchen Nutzen hat in diesem Zusam- menhang die Bestrahlung des gesamten lymphatischen Systems (TLI)?}

Prem atier atienten mit rezidiviertem/refraktärem Hodgkin-Lymphom ( $\mathrm{r} / \mathrm{rHL})$ kann mit einer autologen Stammzelltransplantation (ASCT) geholfen werden. Nach einer IFRT und ASCT werden die meisten Rezidive in Lymphknoten außerhalb des bestrahlten Areals entdeckt. Um solche Rezidive zu verhindern, wurde die TLI entwickelt.

Ärzte vom Memorial Sloan Kettering Cancer Center in New York nutzen die TLI seit 1985 standardmäßig als Teil der Konsolidierung vor der ASCT. Dabei kommt eine integrierte IFRT-TLI zum Einsatz, um die Überlappung der Radiotherapie mit der Hochdosis-Chemotherapie zu minimieren und unnötige Verzögerungen der ASCT zu verhindern. Nun haben sie die Langzeitergebnisse ihrer Patienten zusammengefasst. Zwischen November 1985 und Juli 2008 unterzogen sich 186 zuvor nicht bestrahlte Patienten mit r/rHL dieser Behandlung. Nach einer Salvage-Chemotherapie wurden die Lymphknoten mittels beschleunigter IFRT (18-20 Gy) behandelt, gefolgt von TLI (15-18 Gy) und Hochdosistherapie.

Nach median 57 Monaten betrugen die 5- bzw. 10-Jahres-Überlebensraten 68 bzw. $56 \%$, die ereignisfreien Überlebensraten 62 bzw. $56 \%$ und die kumulativen Inzidenzen von HL-bedingten Todesfällen 21 bzw. $29 \%$.

In einer Multivarianzanalyse war das komplette Ansprechen auf die SalvageChemotherapie unabhängig assoziiert mit einem besseren Gesamt- und ereig- nisfreien Überleben. Primär refraktäre und extranodale Erkrankungen waren dagegen unabhäng voneinander assoziiert mit einem ungünstigen krankheitsspezifischen Überleben.

Bei 8 Patienten entwickelte sich eine kardiale Toxizität vom Schweregrad $\geq 3$, die mit 3 Todesfällen einherging. Sekundäre Malignome entstanden bei 10 Patienten, von diesen starben 5 .

Fazit: Nach einer Salvage-Chemotherapie ist die akzelerierte IFRT, gefolgt von TLI und hochdosierter Chemotherapie, eine wirksame, praktikable und sichere Strategie für Patienten mit $\mathrm{r} / \mathrm{rHL}$. Sie führt zu guten Raten für das LangzeitGesamtüberleben, das ereignisfreie und das krankheitsspezifische Überleben. Das vollständige Ansprechen auf die Salvage-Chemotherapie ist der wichtigste prognostische Faktor. Christina Berndt

Rimner A et al. Accelerated Total Lymphoid Irradiation-containing Salvage Regimen for $\mathrm{Pa}$ tients With Refractory and Relapsed Hodgkin Lymphoma: 20 Years of Experience. Int J Radiat Oncol Biol Phys. 2017;97(5):1066-76. 